\title{
Hall current effects on a magnetic nanofluid layer under temperature gradient
}

\author{
Jaimala Bishnoi ${ }^{1}$ (D) Shubham Kumar ${ }^{1} \cdot$ Reema Singh $^{1}$ (I)
}

Received: 20 August 2020 / Accepted: 20 January 2021 / Published online: 26 January 2021

(C) The Author(s) 2021 OPEN

\begin{abstract}
In the Bénard convection governed nanofluid confined between two horizontal infinite free-free boundaries, the effect of Hall current is investigated when it is subjected to the constant vertical magnetic field. The Buongiorno mathematical model for hydromagnetic flow with Hall currents has been considered. The Brownian motion effects and thermophoresis of nanoparticles have been included in the energy equation. A realistic boundary condition based on zero nanoparticle mass flux at the walls is incorporated for the analysis. The impacts of Brownian motion, convective heat transfer, thermophoresis of nanoparticles and zero mass flux conditions are also deliberated through the behaviour of the related parameters. The parameters representing the model incorporate the consequences of newly introduced physically realistic boundary conditions and Hall currents. For comprehensive physical interpretation the embedded parameters have been plotted and deliberated graphically. It is found that Hall current is responsible to enhances the instability of the system and sets the convection earlier. A rigorous comparison has been made with the existing results. The results are shown graphically and verified numerically.
\end{abstract}

Keywords Nanofluid · Hall current · Brownian motion · Transverse magnetic field

\begin{tabular}{llll} 
Abbreviations & $T$ & Nanofluid temperature (dimensionless) \\
$(\rho c)_{f}$ & Heat capacity of the nanofluid & $(u, v, w)$ & Velocity components (dimensionless) \\
$(\rho c)_{p}$ & Heat capacity of the nanoparticles & $\mathbf{v}$ & Nanofluid velocity (dimensionless) \\
$D_{B}$ & Brownian diffusion coefficient & $(x, y, z)$ & Cartesian coordinate (dimensionless \\
$D_{T}$ & Thermophoresis diffusion coefficient & \multicolumn{2}{c}{ Greek symbols } \\
$\mathbf{H}$ & Magnetic field & $\rho_{p}$ & Nanoparticle density \\
$k$ & Thermal conductivity of nanofluid & $\rho_{f}$ & Base fluid density \\
$L_{e}$ & Lewis number & $\rho_{0}$ & Reference density of nanofluid \\
$N_{A}$ & Modified diffusivity ratio & $\mu_{e}$ & Magnetic permeability \\
$N_{B}$ & Modified particle density increment & $\mu$ & Viscosity \\
$P$ & Pressure & $\phi$ & Dimensionless volume fraction \\
$P_{r}$ & Prandtl number & & \\
$P_{r M}$ & Magnetic Prandtl number & & \\
$Q$ & Nanofluid magnetic number & & \\
$R_{a}$ & Rayleigh number & & \\
$R_{m}$ & Basic-density Rayleigh number & & \\
$R_{n}$ & Concentration Rayleigh number & & \\
$t$ & Time (dimensionless) & &
\end{tabular}

Reema Singh, reemamalik28@gmail.com; Jaimala Bishnoi, jaimalaccsu1@gmail.com; Shubham Kumar, shubhammzn17@gmail.com | 1Department of Mathematics, Chaudhary Charan Singh University, Meerut, UP 250004, India. 


\section{Introduction}

The poor thermal conductivity was a big impediment of the fluids used as cooling stimulants in heat transfer equipment, radiators and electronic cooling systems used in different fields of importance like medicine, defense, transportation etc. Choi and his counterparts [1-3] performed several experiments by suspending various metallic and metal oxide nanoparticles in different types of fluids and inferred that the thermal conductivity of the base fluid can be increased to many folds by adding low concentration of nanosized particles of the materials having higher thermal conductivity than the base fluid. These are two phase fluids where a liquid or gas can act as a host phase and nanosized particles or fibers are included as foreign guests. The particles added are larger than atomic dimensions but small enough (between 1 and $100 \mathrm{~nm}$ ) to exhibit Brownian motion. Another pioneer in the field of nanofluids is Buongiorno [4], who discussed the various slip mechanism important in nanofluids like Brownian diffusion, Magnus effect, inertia, thermophoresis, fluid drainage, diffusionphoresis and gravity setting and recognized that in the absence of turbulent eddies, these are Brownian diffusion and thermophoresis which dominate the other slip mechanisms. It has also been established that the behavior of nanofluid is basically governed by the material of nanoparticles, their volume fraction, size, shape, spatial distribution, $\mathrm{pH}$ value, temperature and the type of the base-fluid. The inclusion of the nanoparticles with a surrounding interfacial layer disturbs the properties of the host fluid in a positive and interesting way and the thermal conductivity of the fluid increases to many folds with an increase in the volume fraction of the nanoparticles. Consequently, the rate of heat transfer is also increased. Thus, the introduction of the nanofluid started a revolution in the fields which were in an urgent need of such type of fluids like transportation, nuclear reactors, medicine, electronics and food [5-8].

Due to temperature-induced differences in buoyancy, free or natural heat convection termed as Bénard convection occurs in fluids. In free convection, the mass transfer process is governed by the variations of the density gradient generating the buoyancy force of the flow. The Bénard convection in nanofluids in the presence of Lorentz force are providing promising results through the progressively ironic and varied technologies used for cancer therapy, solar cell augmentation, pharmacology, flow-meters, pumps, magnetic storage, magnetohydrodynamic generators, accelerators, cooling the electronic equipment, magnetic field sensors, geothermal reservoirs, insulators, heating and ventilation control in building design etc. [9-13].
The Hall effect is nothing but an extension of the Lorentz force, which describes the force exerted on a charged particle moving through a magnetic field. When a sufficiently high strength magnetic field is applied in the direction perpendicular to the electric field, an electric potential is generated across the conductor, which is perpendicular to both the magnetic as well as electric field. This phenomenon is produced due to the induced Lorentz force and is called Hall Effect. It is a measurable transverse voltage discovered by Hall [14]. In fluids specifically in ionized gases or liquids if subjected to a strong magnetic field and even a small electron density, the Hall effects are so effective that these cause the magnetic orientation to change the flow outline of the fluid. Hall current effective IC switch or sensor is exceptionally useful to detect the magnetic field and provides digital signal for on and off in nuclear power reactors, Hall accelerators, flight magnetogasdynamics and magnetohydrodynamic generators. Since nanofluids are performing an observant role in diversified fields, the Hall current effective hydromagnetic flows of nanofluids have been studied by many authors. Recently, Yadav et al. [15] discussed the convection of nanofluids under Hall currents saturated in a Brinkman porous medium. Das et al. [16] studied the Hall effects for natural convection of a nanofluid with thermal radiation. Wahed [17] investigated the flow over a stretching plate embedded in a ferromagnetic fluid suspended with $\mathrm{Fe}_{3} \mathrm{O}_{4}$ nanoparticles in the presence of hall current, nonlinear thermal radiation and joule heating. Abbasi et al. [18] investigated the effect of Hall currents on a nanofluid with variable thermal conductivity. Shah et al. [19] discussed the flow on a micropolar nanofluid saturated between two horizontal parallel plates in a rotating reference frame under the influence of Hall current. Krishna and Chamkha [20] analyzed the Hall effects on the flow of a water-based nanofluid using Galerkin optimal homotopy asymptotic method. Further, ion slip and hall effect is seen by Krishna and Chamkha for $\mathrm{Ag}$ and $\mathrm{TiO}_{2}$ nanofluids using perturbation technique [21]. Shah et al. [22] studied the impact of Hall effect in the presence of Cattaneo-Christov heat flux and rotation on the squeezing 3-D flow of nanofluid between parallel plates. Sharma and Gupta [23] discussed the thermal instability of nanofluids under Hall currents subjected to the local thermal non-equilibrium (LTNE) effects.

The above-mentioned literature does not conduct the Hall currents in nanofluid with passive management of nanoparticles at the boundary walls. The present study focuses to explore the role of Hall current to the thermal convection of nanofluid under constant transversal magnetic field considering the realistic boundary conditions motivated by the prominence passive management of temperature at the boundaries suggested by Stefan [24]. The Galerkin-type weighted residuals method is used. To 
see the flow pattern of nanofluid, comparative graphs are also given. Using MATLAB software, the stationary convection is discussed analytically as well as graphically.

\section{Mathematical modeling}

Consider a layer of electrically conducting incompressible fluid comprised with nanoparticles. A Cartesian co-ordinate system $(x, y, z)$ is chosen where $z$ axis is taken at right angle to boundaries. The fluid layer is lying between infinitely extended horizontal plates situated at $z^{*}=0$ and $z^{*}=L$. The system is heated from below such that the temperature $T_{0}^{*}$ at the lower boundary, is greater than the temperature $T_{1}^{*}$ at the upper boundary. Further consider that the transversal vertical magnetic field $\mathbf{H}^{*}=\left(0,0, H_{0}^{*}\right)$ acts on the system.

The mathematical equations relevant to the physical configuration are taken from Yadav et al. $[15,25]$. The mass-conservation equation for the nanofluid and the nanoparticle are respectively taken as

$\nabla \cdot \mathbf{v}^{*}=0$

and

$\left(\frac{\partial}{\partial t^{*}}+\left(\mathbf{v}^{*} \cdot \nabla\right)\right) \phi^{*}=D_{B} \nabla^{*^{2}} \phi^{*}+\left(\frac{D_{T}}{T_{1}^{*}}\right) \nabla^{*^{2}} T^{*^{2}}$,

The energy equation and momentum conservation equation are considered as where $\mathbf{v}^{*}$ is the nanofluid velocity, $\phi^{*}$ is the volume fraction of nanoparticles ( $\phi_{0}^{*}$ is the reference value), $t^{*}$ is the time, $p^{*}$ is the pressure, $\rho$ is the density of the base fluid, $\rho_{p}$ is the density of nanoparticles, $\rho_{0}$ is the reference density of nanofluid, $\mathbf{g}$ is the gravitational acceleration, $(\rho c)_{f}$ is the heat capacity of the nanofluid, $(\rho c)_{p}$ is the heat capacity of the nanoparticles, $D_{B}$ is the Brownian diffusion coefficient, $D_{T}$ is the thermophoretic diffusion coefficient, $T_{1}^{*}$ is the reference temperature, $k$ is the thermal conductivity, $\beta$ is thermal expansions coefficient, $\mu$ is viscosity,$\eta$ is electrical resistivity, $\xi$ is the velocity of light, $e$ is charge of electron and $N$ is the electron number density.

On the boundaries, the temperature is taken as constant while the nanoparticle flux is assumed to be zero [25] and therefore, we assume

$W^{*}=0, T^{*}=T_{0}^{*}, D_{B} \frac{d \phi^{*}}{d z^{*}}+\frac{D_{T}}{T_{1}^{*}} \frac{d T^{*}}{d z^{*}}=0$ at $z^{*}=0$,

$W^{*}=0, T^{*}=T_{1}^{*}, D_{B} \frac{d \phi^{*}}{d z^{*}}+\frac{D_{T}}{T_{1}^{*}} \frac{d T^{*}}{d z^{*}}=0$ at $z^{*}=L$,

Introducing non-dimensional parameters as

$$
\begin{gathered}
(x, y, z)=\frac{\left(x^{*}, y^{*}, z^{*}\right)}{L}, \quad(u, v, w)=\frac{\left(u^{*}, v^{*}, w^{*}\right)}{\alpha} L, \quad\left(H_{x}, H_{y}, H_{z}\right)=\frac{\left(H_{x}^{*}, H_{y}^{*}, H_{z}^{*}\right)}{H_{0}^{*}}, \\
t=\frac{t^{*}}{L^{2}} \alpha, \quad p=\frac{p^{*}}{\mu \alpha} L^{2}, \quad \phi=\frac{\phi^{*}-\phi_{0}^{*}}{\phi_{0}^{*}}, \quad T=\frac{T^{*}-T_{1}^{*}}{T_{0}^{*}-T_{1}^{*}},
\end{gathered}
$$

Equations (1)-(6) are obtained in non-dimensional form

$$
\begin{aligned}
(\rho c)_{f}\left(\frac{\partial}{\partial t^{*}}+\left(\mathbf{v}^{*} \cdot \nabla^{*}\right)\right) T^{*} & =k \nabla^{*^{2}} T^{*}+(\rho c)_{p}\left[D_{B} \nabla^{*} \phi^{*} \cdot \nabla^{*} T^{*}+\frac{D_{T}}{T_{1}^{*}} \nabla^{*} T^{*} \cdot \nabla^{*} T^{*}\right], \\
\rho_{0}\left(\frac{\partial}{\partial t^{*}}+\left(\mathbf{v}^{*} \cdot \nabla\right)\right) \mathbf{v}^{*}= & -\nabla^{*} p^{*}+\mu \nabla^{*^{2}} \mathbf{v}^{*}+\left[\phi^{*} \rho_{p}+\left(1-\phi^{*}\right) \rho_{0}\left\{1-\beta\left(T^{*}-T_{1}^{*}\right)\right\}\right] \mathbf{g} \\
& +\frac{\mu_{e}}{4 \pi}\left(\nabla^{*} \times \mathbf{H}^{*}\right) \times \mathbf{H}^{*} .
\end{aligned}
$$

The modified Maxwell equations under the influence of Hall currents are taken as

$$
\begin{aligned}
\left(\frac{\partial}{\partial t^{*}}+\left(\mathbf{v}^{*} \cdot \nabla^{*}\right)\right) \mathbf{H}^{*}= & \left(\mathbf{H}^{*} \cdot \nabla^{*}\right) \mathbf{v}^{*}+\eta \nabla^{*^{2}} \mathbf{H}^{*} \\
& -\frac{\xi}{4 \pi e N} \nabla^{*} \times\left[\left(\nabla^{*} \times \mathbf{H}^{*}\right) \times \mathbf{H}^{*}\right],
\end{aligned}
$$

$\nabla^{*} \cdot \mathbf{H}^{*}=0$, as follows:

$$
\begin{aligned}
& \nabla \cdot \mathbf{v}=0 \\
& \begin{aligned}
\frac{1}{P_{r}}\left[\frac{\partial}{\partial t}+(\mathbf{v} \cdot \nabla)\right] \mathbf{v}= & -\nabla p+\nabla^{2} \mathbf{v}-R_{m} \hat{\mathbf{e}}_{z}+R_{a} T \hat{\mathbf{e}}_{z} \\
& -R_{n} \phi \hat{\mathbf{e}}_{z}+Q \frac{P_{r}}{P_{r M}}(\nabla \times \mathbf{H}) \times \mathbf{H},
\end{aligned}
\end{aligned}
$$


$\left[\frac{\partial}{\partial t}+(\mathbf{v} \cdot \nabla)\right] T=\nabla^{2} T+\frac{N_{B}}{L_{e}}(\nabla T \cdot \nabla \phi)+\frac{N_{A} N_{B}}{L_{e}}(\nabla T \cdot \nabla T)$,

$\left[\frac{\partial}{\partial t}+(\mathbf{v} \cdot \nabla)\right] \phi=\frac{N_{A}}{L_{e}} \nabla^{2} T+\frac{1}{L_{e}} \nabla^{2} \phi$

$\left[\frac{\partial}{\partial t}+(\mathbf{v} \cdot \nabla)\right] \mathbf{H}=(\mathbf{H} \cdot \nabla) \mathbf{v}+\frac{P_{r}}{P_{r M}} \nabla^{2} \mathbf{H}-M \frac{P_{r}}{P_{r M}} \nabla \times[(\nabla \times \mathbf{H}) \times \mathbf{H}]$,

$\nabla \cdot \mathbf{H}=0$.

The non-dimensional form of boundary conditions is obtained as

$\mathrm{W}=0, \mathrm{~T}=1, \frac{d \phi}{d z}+N_{A} \frac{d T}{d z}=0$ at $z=0$,

$\mathrm{w}=0, \mathrm{~T}=0, \frac{d \phi}{d z}+N_{A} \frac{d T}{d z}=0$ at $z=1$.

In the above process the following non-dimensional parameters are obtained:

$L_{e}=\frac{\alpha}{D_{\beta}}$ (Nanofluid Lewis number),

$N_{A}=\frac{B_{T}\left(T_{0}^{*}-T_{1}^{*}\right)}{D_{B} T_{T}^{*} \phi_{0}^{*}}$ (Modified diffusivity ratio number),

$N_{B}=\frac{(\rho c)_{p} \phi_{0}^{*}}{(\rho c)_{f}}$ (Modified particle density increment number),

$P_{r}=\frac{\mu}{\rho_{0} \alpha}$ (Nanofluid Prandtl number),

$P_{r M}=\frac{\mu}{\rho_{0}}$ (Magnetic Prandtl number),

$R_{a}=\frac{\rho g \beta{ }^{3}\left(T_{0}^{*}-T_{1}^{*}\right)}{\mu \alpha}$ (Rayleigh number),

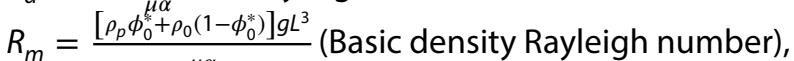

$R_{n}=\frac{\left(\rho_{p}-\rho_{0}\right) \phi_{0}^{\mu \alpha} g L^{3}}{(C o n c e n t r a t i o n ~ R a y l e i g h ~ n u m b e r), ~}$

$Q=\frac{\mu_{e} H_{0}^{* 2} L^{\mu^{\alpha}}}{4 \pi \rho_{0} v \eta}$ (Nanofluid magnetic number),

and $M=\frac{H_{0}^{*} \xi}{4 \pi e N \eta}$ (Hall Current number).

Following [4] density of nanoparticles is assumed to be greater than the density of the base fluid $\left(\rho_{p}>\rho\right)$. Thus, throughout the analysis to follow $R_{n}$ is taken as positive.

\subsection{Conduction state}

Assume that the basic state is time independent and is given by

$\mathbf{v}=0, \quad T=T_{b}(z), \quad \phi=\phi_{b}(z), \rho=\rho_{b}(z), p=p_{b}(z), \mathbf{H}=\hat{\mathbf{e}}_{z}$.

Substituting (18) in the flow governing equations and performing certain mathematical calculations, we get $\frac{d \phi_{b}}{d z}+N_{A} \frac{d T_{b}}{d z}=0$

and

$\frac{d^{2} T_{b}}{d z^{2}}=0$

Equations (19) and (20) provide the following steady state solutions compatible with the boundary conditions:

$T_{b}=1-z$,

$\phi_{b}=\phi_{0}+z N_{A}$.

\subsection{Perturbed state}

Imposing the space and time dependent perturbations onto the basic state, we get

$v=v^{\prime}, \quad T=T_{b}(z)+T^{\prime}, \quad \phi=\phi_{b}(z)+\phi^{\prime}, \quad \rho=\rho_{b}(z)+\rho^{\prime}$,
$\mathbf{H}=\hat{\mathbf{e}}_{z}+\mathbf{H}^{\prime}$.

where prime $\left({ }^{\prime}\right)$ denotes the perturbations /disturbances.

Substituting Eq. (23) into Eqs. (10)-(15) and under linear stability theory neglecting the product of prime quantities, we get

$\nabla \cdot \mathbf{v}^{\prime}=0$

$\frac{1}{P_{r}} \frac{\partial \mathbf{v}^{\prime}}{\partial t}=-\nabla p^{\prime}+\nabla^{2} \mathbf{v}^{\prime}+R_{a} T^{\prime} \hat{\mathbf{e}}_{z}-R_{n} \phi_{z}^{\prime} \hat{\mathbf{e}}_{z}+Q \frac{P_{r}}{P_{r M}}\left[\left(\nabla \times \mathbf{H}^{\prime}\right) \times \hat{\mathbf{e}}_{z}\right]$,

$\frac{\partial T^{\prime}}{\partial t}-w^{\prime}=\nabla^{2} T^{\prime}+\frac{N_{B}}{L_{e}}\left[N_{A} \frac{\partial T^{\prime}}{\partial z}-\frac{\partial \phi^{\prime}}{\partial z}\right]-2 \frac{N_{A} N_{B}}{L_{e}} \frac{\partial T^{\prime}}{\partial z}$,

$\frac{\partial \phi^{\prime}}{\partial t}+N_{A} w^{\prime}=\frac{1}{L_{e}} \nabla^{2} \phi^{\prime}+\frac{N_{A}}{L_{e}} \nabla^{2} T^{\prime}$

$\frac{\partial \mathbf{H}^{\prime}}{\partial t}=\frac{\partial w^{\prime}}{\partial z} \hat{\mathbf{e}}_{z}+\frac{P_{r}}{P_{r M}} \nabla^{2} \mathbf{H}^{\prime}-M \frac{P_{r}}{P_{r M}} \nabla \times\left[\left(\nabla \times \mathbf{H}^{\prime}\right) \times \hat{\mathbf{e}}_{z}\right]$,

$\nabla \cdot \mathbf{H}^{\prime}=0$

Operate $\hat{\mathbf{e}}_{\mathrm{z}^{*}}$ curl curl, on Eq. (28), and use the identity curl curl = grad (div) $-\nabla^{2}$. The resulting equation and Eq. (29), help to provide the $z$-component of the momentum equation given by Eq. (25) in the following form:

$\left[\nabla^{4}-\frac{1}{P_{r}} \frac{\partial}{\partial t} \nabla^{2}\right] w^{\prime}+R_{a} \nabla_{p}^{2} T^{\prime}-R_{n} \nabla_{p}^{2} \phi^{\prime}+Q \frac{P_{r}}{P_{r M}}\left(\frac{\partial \mathbf{H}^{\prime}}{\partial z}\right)=0$,

Solving Eq. (28) we get 
$\frac{\partial H_{x}^{\prime}}{\partial t}=\frac{P_{r}}{P_{r M}} \nabla^{2} H_{x}^{\prime}-M \frac{P_{r}}{P_{r M}} \frac{\partial \zeta_{x}^{\prime}}{\partial z}$

$\frac{\partial H_{y}^{\prime}}{\partial t}=\frac{P_{r}}{P_{r M}} \nabla^{2} H_{y}^{\prime}-M \frac{P_{r}}{P_{r M}} \frac{\partial \zeta_{y}^{\prime}}{\partial z}$

$\frac{\partial H_{z}^{\prime}}{\partial t}=\frac{P_{r}}{P_{r M}} \nabla^{2} H_{z}^{\prime}-M \frac{P_{r}}{P_{r M}} \frac{\partial \zeta_{z}^{\prime}}{\partial z}$.

Using Eqs. (31) to (33), we get

$\left(\nabla^{4}-\frac{1}{P_{r}} \nabla^{2} \frac{\partial}{\partial t}\right) \Gamma_{2} w^{\prime}+R_{a} \nabla_{p}^{2} \Gamma_{2} T^{\prime}-R_{n} \nabla_{p}^{2} \Gamma_{2} \phi^{\prime}$

$-Q \frac{P_{r}}{P_{r M}} \nabla^{2} \Gamma_{1} \frac{\partial^{2} w^{\prime}}{\partial z^{2}}=0$,

where the operators $\Gamma_{1}$ and $\Gamma_{2}$ are given by

$\Gamma_{1}=\left(\frac{P_{r}}{P_{r M}} \nabla^{2}-\frac{\partial}{\partial t}\right)$,

$\Gamma_{2}=\left[\Gamma_{1}^{2}+\left(M \frac{P_{r}}{P_{r M}}\right)^{2} \nabla^{2} \frac{\partial^{2}}{\partial z^{2}}\right]$

\section{Linear stability analysis}

Assume the small perturbations in the form of wave and decompose them as follows:

$\left(w^{\prime}, T^{\prime}, \phi^{\prime}\right)=[W(z), \Theta(z), \Phi(z)] \exp \left[i k_{x} x+i k_{y} y+\sigma t\right]$,

where $k_{x}$ and $k_{y}$ are the wave numbers along the $x$ and $y$ directions respectively, and $a=\sqrt{k_{x}^{2}+k_{y}^{2}}$ is the resultant wave number.
Using (37) in Eqs. (26), (27) and (34) the resulting equations governing the stability or instability of the system are given by

$W+\left[D^{2}-a^{2}-\frac{N_{A} N_{B}}{L_{e}}-\sigma\right] \Theta-\frac{N_{B}}{L_{e}} D \Phi=0$,

$N_{A} W-\frac{N_{A}}{L_{e}}\left[D^{2}-a^{2}\right] \Theta+\left[\sigma-\frac{1}{L_{e}}\left(D^{2}-a^{2}\right)\right] \Phi=0$,

$\left(D^{2}-a^{2}\right)\left[\left(D^{2}-a^{2}\right)-\frac{\sigma}{P_{r}}\right] \Gamma_{4} W-R_{a} a^{2} \Gamma_{4} \Theta$

$+R_{n} a^{2} \Gamma_{4} \Phi-\left[Q \frac{P_{r}}{P_{r M}}\left(D^{2}-a^{2}\right)\right] \Gamma_{3} D^{2} W=0$,

where

$\Gamma_{3}=\left[\frac{P_{r}}{P_{r M}}\left(D^{2}-a^{2}\right)-\sigma\right]$,

$\Gamma_{4}=\left[\left(\Gamma_{3}\right)^{2}+\left(M \frac{P_{r}}{P_{r M}}\right)^{2}\left(D^{2}-a^{2}\right) D^{2}\right]$.

Use Galerkin weighted residual technique and assume the approximate solutions compatible with the boundary conditions as

$W=W_{0} \operatorname{Sin} \pi z, \quad T=\Theta_{0} \operatorname{Sin} \pi z, \quad \Phi=-N_{A} \Phi_{0} \operatorname{Sin} \pi z$.

Substituting (43) in Eq. (38)-(40), and integrating the resulting equations with respect to $z$ from $z=0$ to $z=1$, we have

$W_{0}-(J+\sigma) \Theta_{0}=0$,

$W_{0}+\frac{J}{L_{e}} \Theta_{0}-\left(\sigma+\frac{J}{L_{e}}\right) \Phi_{0}=0$,

$\left[\frac{P_{r}^{2}}{P_{r M}^{2}} J^{4}+\frac{\sigma}{P_{r M}} \jmath^{3}+\frac{\sigma^{3}}{P_{r}}+\frac{\sigma^{2}}{P_{r M}} J+\frac{M^{2} \sigma P_{r} \pi^{2} \jmath}{P_{r M}^{2}}+\sigma^{2} \jmath^{2}+\frac{2 P_{r} \sigma \jmath^{3}}{P_{r M}}+\frac{M^{2} P_{r}^{2} \pi^{2} \jmath^{3}}{P_{r M}^{2}}+\frac{Q P_{r}}{P_{r M}}\left(\frac{P_{r} \pi^{2} \jmath^{2}}{P_{r M}}+\sigma \pi^{2} \jmath\right)\right] W_{0}$

$-\left(R_{a} a^{2} \Gamma_{5}\right) \Theta_{0}-\left(N_{A} R_{n} a^{2} \Gamma_{5}\right) \Phi_{0}=0$, 
where

$\Gamma_{5}=\left\{\frac{P_{r}^{2} \jmath^{2}}{P_{r M}^{2}}+\sigma^{2}+\frac{\left.2 \sigma P_{r}\right\lrcorner}{P_{r M}}+\frac{\left.M^{2} \pi P_{r}^{2}\right\lrcorner}{P_{r M}^{2}}\right\}$ and $J=\pi^{2}+a^{2}$.

For a non-trivial solution of the system of equations in $W_{0}$ $\Theta_{0}$ and $\Phi_{0}$ given by (44)-(46), the determinant of coefficients should be zero i.e.
The critical wave number for stationary convection may be obtained from Eq. (51). Here it has been confirmed that the Hall current and magnetic field register their effects by modifying the critical wave number which has been obtained for a fluid without magnetic field.

To investigate the stability or instability of the flow we calculate the variations in Rayleigh number $R_{a}$ against the magnetic number $Q$, Hall current parameter $M$, Lewis

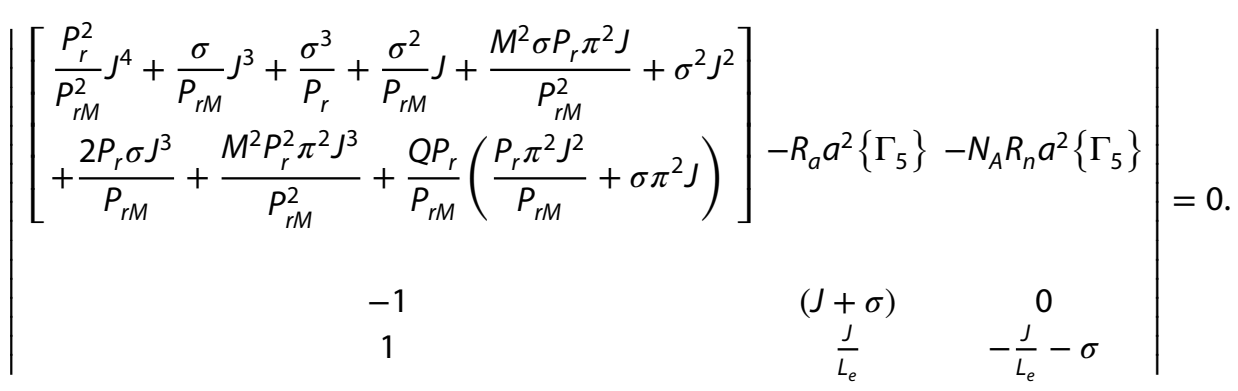

\subsection{Stationary Convection}

In order to analyze stationary convection at the marginal state we put $\sigma=0$ in Eq. (48) and get the Rayleigh number as

$R_{a}=\frac{J^{2}\left(J^{2}+Q \pi^{2}+M^{2} \pi^{2} J\right)}{\left(J+M^{2} \pi\right) a^{2}}-N_{A} R_{n}\left(1+L_{e}\right)$.

Substituting $a^{2}=\pi^{2} x$ in $J$, Eq. (49) provides

$R_{a}=\frac{\pi^{3}(1+x)^{2}\left[\pi^{2}(1+x)^{2}+Q+M^{2} \pi(1+x)\right]}{\left[(1+x) \pi+M^{2}\right] x}-N_{A} R_{n}\left(1+L_{e}\right)$.

It is important to mention that the Rayleigh number given by (49) is governed by the parameters introduced due to Hall currents. All the parameters are also modified according to the new boundary conditions. Equation (50) says that if there is a regular fluid $\left(N_{A}=0\right)$, then the Rayleigh number in the presence of magnetic field is increased showing that the presence of nanoparticles will exaggerate the convection earlier.

Further, if we put $N_{A}=0, R_{n}=0, L e=0$ and $M=0$, the Rayleigh number for the regular fluids [27] has been recovered.

In order to obtain the critical wave number, we put $\frac{d R_{a}}{d x}=0$. We get the following fourth-degree equation in $x_{c}\left(=\pi x^{\frac{1}{2}}\right)$ :

$$
\begin{aligned}
&\left(2 \pi^{3}\right) x_{c}^{4}+\left(5 \pi^{3}+4 M^{2} \pi^{2}\right) x_{c}^{3}+\left(3 \pi^{3}+6 M^{2} \pi^{2}+2 M^{4} \pi\right) x_{c}^{2} \\
&-\left(\pi^{3}-M^{4} \pi-Q M^{2}+\pi Q\right) x_{c}-\left(\pi^{3}+2 M^{2} \pi^{2}+M^{4} \pi+\pi Q+Q M^{2}\right)=0 .
\end{aligned}
$$

number $L_{e^{\prime}}$ modified diffusivity ratio number $N_{A}$ and concentration Rayleigh number $R_{n}$.

Calculating the derivative of $R_{a}$ with respect to $Q$ we get

which is clearly positive i.e., the Rayleigh number for stationary convection is increasing on increasing the magnetic field parameter $Q$. Thus, the magnetic field helps to suppress the instability of the nanofluid.

$\frac{d R_{a}}{d M}=\frac{2 M \pi\left(a^{2}+\pi^{2}\right)^{2}\left\{(\pi-1)\left(a^{2}+\pi^{2}\right)^{2}-Q \pi^{2}\right\}}{\left(a^{2}+\pi^{2}+M^{2} \pi\right) a^{2}}$

conveys that the hall current parameter $M$ stabilizes the system only for $Q<\frac{(\pi-1)\left(a^{2}+\pi^{2}\right)^{2}}{\pi^{2}}$ and when $Q>\frac{(\pi-1)\left(a^{2}+\pi^{2}\right)^{2}}{\pi^{2}}$ the system is unstable with increasing $M$.

$\frac{d R_{a}}{d L_{e}}=-N_{A} R_{n}$

predicts that the nanofluid Lewis number is crashing the stability of the system.

From Eq. (49), we get

$\frac{d R_{a}}{d N_{A}}=-\left(1+L_{e}\right) R_{n}$ $\frac{d R_{a}}{d Q}=\frac{\left(a^{2}+\pi^{2}\right)^{3} \pi^{2}}{\left(a^{2}+\pi^{2}+M^{2} \pi\right) a^{2}}$ 
and

$\frac{d R_{a}}{d R_{n}}=-\left(1+L_{e}\right) N_{A}$

Equations (55) and (56) show that $N_{A}$ and $R_{n}$ both individually have destabilizing effect.

\subsection{Oscillatory Convection}

Let us assume that at the marginal state, the disturbances provoke or die as oscillatory modes, i.e., $\sigma=i \omega,(\omega>0)$, then from (48) Rayleigh number is obtained as

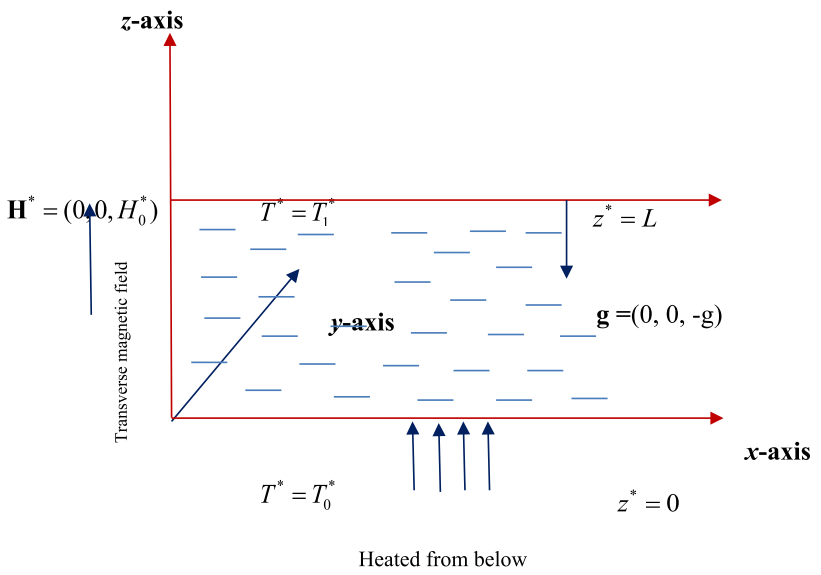

$R_{a}=\frac{(A C+B D)+i \omega(B C-A D)}{\left(C^{2}+D^{2}\right)}$

where $A, B, C$ and $D$ are given as

Fig. 1 Physical configuration

$$
\begin{aligned}
& A=\left\{\begin{array}{l}
\frac{P_{r}^{2} J^{6}}{P_{r M}^{2} L_{e}}-\frac{\omega^{2} J^{3}}{P_{r M} L_{e}}-\frac{\omega^{2} J^{4}}{L_{e}}+\frac{M^{2} P_{r}^{2} \pi^{2} J^{5}}{L_{e}}+\frac{Q P_{r}^{2} \pi^{2} J^{4}}{P_{r M}^{2} L_{e}}-\frac{J^{4} \omega^{2}}{P_{r M} L_{e}}+\frac{J \omega^{4}}{P_{r} L_{e}}-\frac{M^{2} P_{r} \pi^{2} J^{2} \omega^{2}}{P_{r M}^{2} L_{e}} \\
-\frac{2 \omega^{2} P_{r} J^{4}}{P_{r M} L_{e}}-\frac{Q P_{r} \pi^{2} J^{2} \omega^{2}}{P_{r M} L_{e}}-\frac{J^{4} \omega^{2}}{P_{r M}}+\frac{J \omega^{4}}{P_{r}}-\frac{M^{2} P_{r} \pi^{2} J^{2} \omega^{2}}{P_{r M}^{2}}-\frac{2 \omega^{2} P_{r} J^{4}}{P_{r M}}-\frac{Q P_{r} \pi^{2} J^{2} \omega^{2}}{P_{r M}} \\
-\frac{P_{r}^{2} J^{4} \omega^{2}}{P_{r M}^{2}}+\frac{J \omega^{4}}{P_{r}}+\omega^{4} J-\frac{M^{2} P_{r}^{2} \pi^{2} \jmath^{3} \omega^{2}}{P_{r}^{2}}-\frac{Q P_{r}^{2} \pi^{2} J^{2} \omega^{2}}{P_{r M}^{2}}-\frac{N_{A} R_{n} P_{r}^{2} J^{3} a^{2}}{P_{r M}^{2} L_{e}}-\frac{N_{A} R_{n} J \omega^{2} a^{2}}{L_{e}} \\
-\frac{N_{A} R_{n} M^{2} P_{r}^{2} \pi^{2} J^{2} a^{2}}{P_{r M}^{2} L_{e}}-\frac{N_{A} R_{n} P_{r}^{2} J^{3} a^{2}}{P_{r M}^{2}}+N_{A} R_{n} a^{2} \omega^{2} J-\frac{N_{A} R_{n} M^{2} P_{r}^{2} \pi J^{2} a^{2}}{P_{r M}^{2}}+\frac{2 N_{A} R_{n} P_{r} \omega^{2} J a^{2}}{P_{r M}}
\end{array}\right\},
\end{aligned}
$$

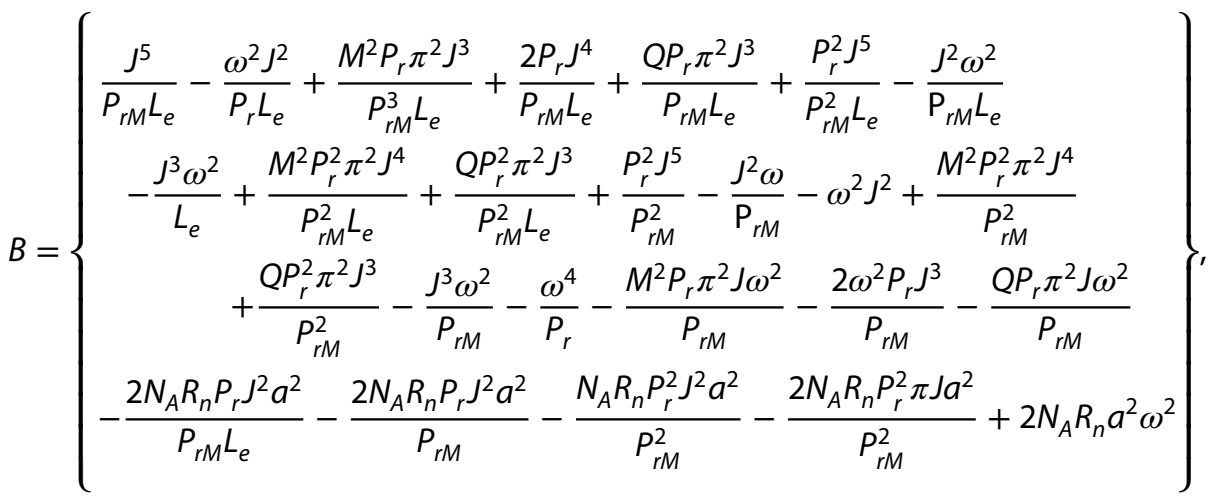

$C=\left\{\frac{P_{r}^{2} \jmath^{3}}{P_{r M}^{2} L_{e}}-\frac{\omega^{2} \jmath}{L_{e}}+\frac{M^{2} P_{r}^{2} \pi^{2} \jmath^{2}}{P_{r M}^{2} L_{e}}+\frac{2 \omega^{2} P_{r} \jmath}{P_{r M}}\right\} a^{2}$

and

$D=\left\{\frac{P_{r}^{2} \jmath^{2}}{P_{r M}^{2}}-\omega^{2}+\frac{M^{2} P_{r}^{2} \pi J}{P_{r M}^{2}}-\frac{2 P_{r} \jmath^{2}}{P_{r M} L_{e}}\right\} a^{2}$.
Since the Rayleigh number $R_{a}$ is always a real physical quantity, Eq. (57) says that we should necessarily have

$$
B C-A D=0
$$

or

$F_{1} \lambda^{3}+F_{2} \lambda^{2}+F_{3} \lambda+F_{4}=0$,

where $\lambda=\omega^{2}$, 


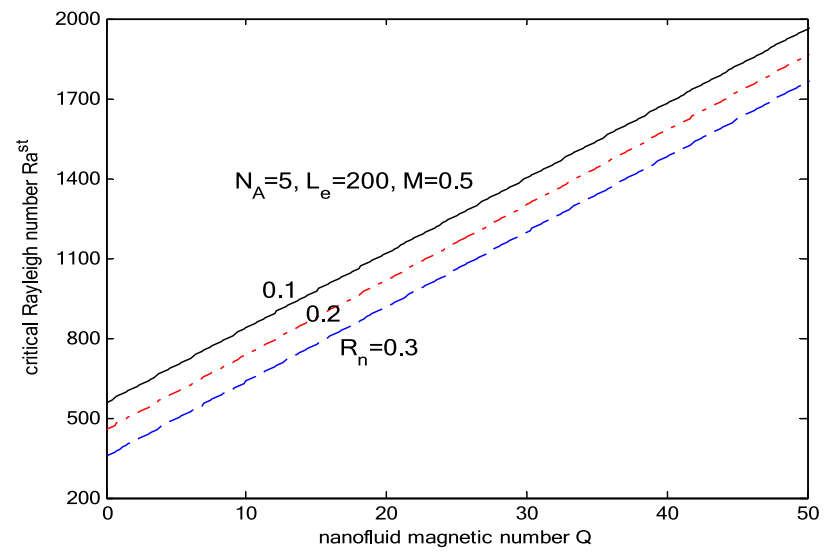

Fig. $2 R_{a}^{\text {st }}$ vs. $Q$ for concentration Rayleigh number $R_{n}$

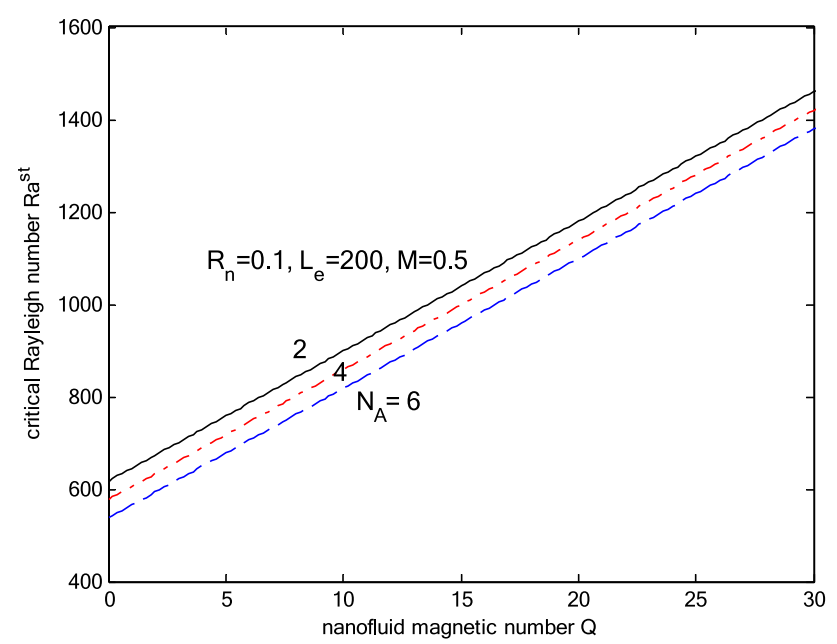

Fig. $3 R_{a}^{\text {st }}$ vs. $Q$ for modified diffusivity ratio $N_{A}$

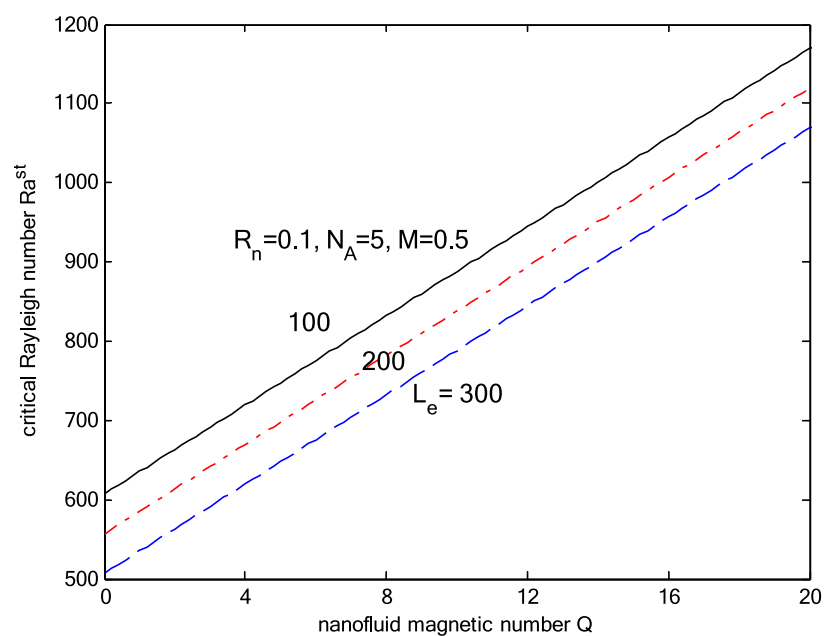

Fig. $4 R_{a}^{s t}$ vs. $Q$ for Lewis number $L_{e}$

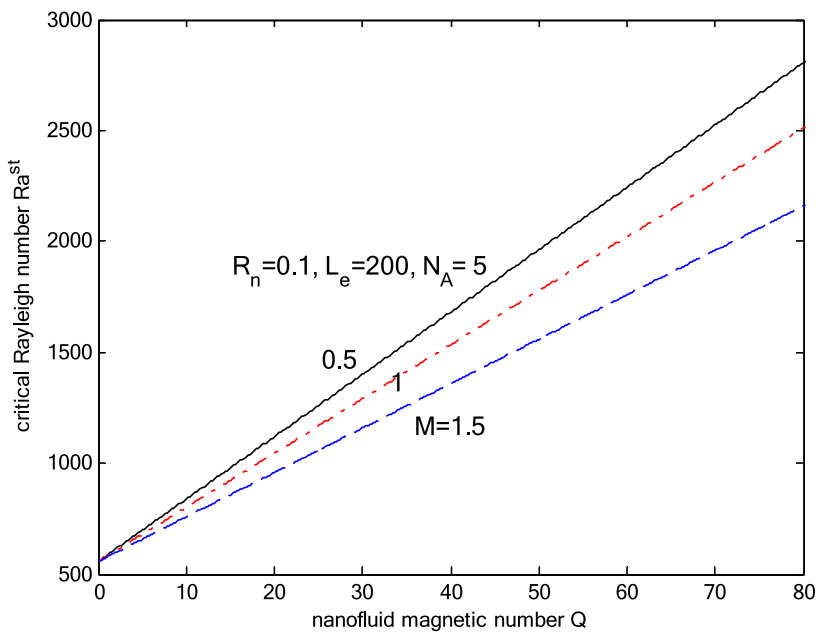

Fig. $5 R_{a}^{\text {st }}$ vs. $Q$ for Hall current parameter $M$

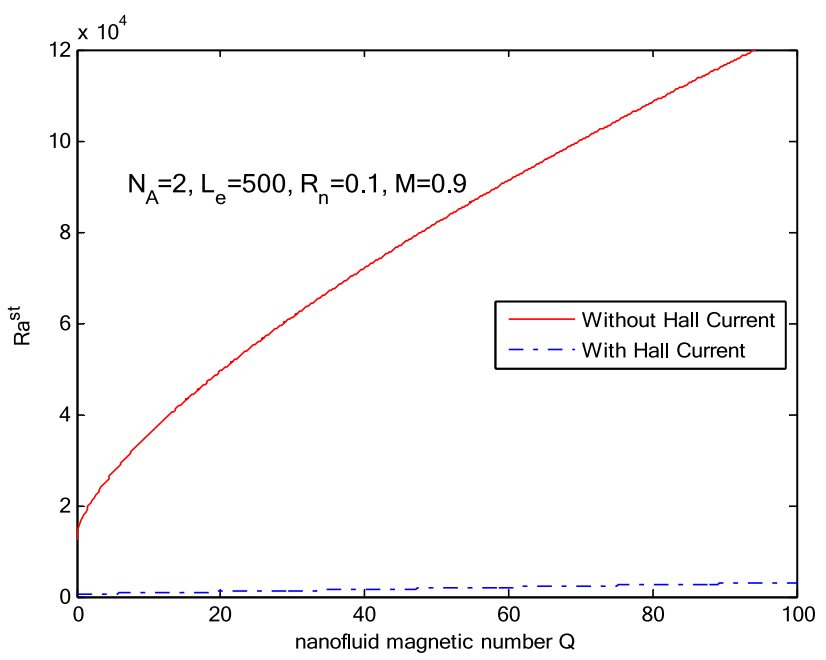

Fig. 6 Comparative study of convection with and without Hall current

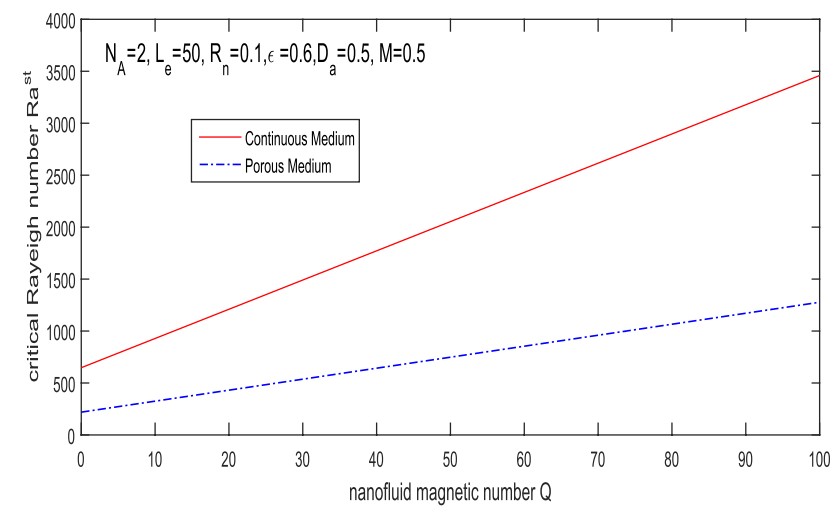

Fig. 7 Comparative graph for convection in a porous medium and a continuous medium

\section{SN Applied Sciences}




$$
\begin{aligned}
& F_{1}=\frac{J a^{2}}{P_{r} P_{r M} L_{e}}\left\{P_{r} P_{r M} L_{e}+2 P_{r M} L_{e}+2 P_{r M}-2 P_{r}^{2}\right\}, \\
& F_{2}=\frac{J a^{2}}{P_{r} P_{r M}^{2} L_{e}^{2}}\left\{\begin{array}{c}
-2 P_{r}^{2} L_{e} J^{2}-2 M^{2} P_{r}^{2} L_{e} \pi^{2} J-M^{2} P_{r}^{2} L_{e} \pi J+4 P_{r}^{2} L_{e} J-2 P_{r}^{2} L_{e} J^{2}-2 P_{r}^{3} L_{e} J^{2} \\
-2 M^{2} P_{r}^{3} L_{e} \pi J-2 P_{r}^{2} L_{e}^{2} J^{2}-2 P_{r}^{2} L_{e}^{2} J^{3}-2 M^{2} P_{r}^{3} L_{e}^{2} \pi^{2} J-5 P_{r}^{3} L_{e}^{2} J^{3}-3 Q P_{r}^{3} L_{e}^{2} \pi^{2} J \\
-M^{2} P_{r}^{2} L_{e}^{2} \pi^{2} J-P_{r}^{3} L_{e}^{2} J^{2}-M^{2} P_{r}^{3} L_{e}^{2} \pi J-M^{2} P_{r}^{3} L_{e}^{2} \pi^{2} J^{2}+P_{r} J^{2}+M^{2} P_{r M} P_{r}^{2} L_{e} \pi J \\
+2 P_{r}^{2} P_{r M} L_{e} J^{2}-4 P_{r}^{2} P_{r M} L_{e}^{2} J^{2}+6 N_{A} R_{n} P_{r}^{2} P_{r M} L_{e} a^{2}-P_{r} P_{r M} L_{e}^{2} J^{3}-2 P_{r}^{2} P_{r M} L_{e}^{2} J^{3} \\
-Q P_{r}^{2} P_{r M} L_{e}^{2} \pi^{2} J-P_{r M}^{2} J^{2}+P_{r} P_{r M}^{2} J^{3}-3 N_{A} R_{n} P_{r} P_{r M}^{2} L_{e} a^{2}+N_{A} R_{n} P_{r} P_{r M}^{2} L_{e}^{2} a^{2}
\end{array}\right\} .
\end{aligned}
$$

The lengthy expressions of $F_{3}$ and $F_{4}$ are not required in the analysis of over-stability, so we can skip them here.

It is to be noted that Eq. (63) is a cubic equation in $\lambda\left(=\omega^{2}\right)$, therefore it has three roots /values which are necessarily to be real and positive. The sum of the roots of Eq. (63) is $-F_{2} / F_{1}$, therefore for oscillatory convection to occur, either $F_{1}$ or $F_{2}$ has to be negative. If both of them are of the same symbol positive or negative, the chances of oscillatory convection are excluded.

It is absorbing to note that following Buongiorno [4] and Yadav et al. [15], the parameters are assigned the following values relevant for alumina water nanofluid suspensions: number decreases with the increasing concentration Rayleigh number. Thus, the parameter $R_{n}$ has destabilizing effect. It is simply a realistic situation because the Brownian motion of nanoparticles increases as volumetric ratio of nanoparticles increases.

In Fig. 3, $R_{a}$ vs. $Q$ curves are drawn for different values of modified diffusivity ratio $N_{A}$ for fixed $L_{e}=200, R_{n}=0.1$ and $M=0.5$. In analogy with the concentration Rayleigh number the increasing values of $N_{A}$ are decreasing the value of Rayleigh number $R_{a}$. Hence, the modified diffusivity ratio $N_{A}$ advances the onset of convection.

The effect of nanofluid Lewis number $L_{e}$ has been

$\phi_{0}^{*}=0.01, \quad \rho_{f 0}=1000 \mathrm{~kg} / \mathrm{m}^{3}, \quad \mu=10^{-3}$ Pas $, \quad \rho_{p}=4 \times 10^{3} \mathrm{~kg} / \mathrm{m}^{3}, D_{B}=4 \times 10^{-11} \mathrm{~m}^{2} / \mathrm{s}$

$D_{T}=6 \times 10^{-11} \mathrm{~m}^{2} / \mathrm{s}, \quad \alpha_{f}=2.0 \times 10^{-7} \mathrm{~m}^{2} / \mathrm{s}, \quad(\rho c)_{p}=3.1 \times 10^{-6} \mathrm{~J} / \mathrm{m}^{3}$

$(\rho c)_{f}=4 \times 10^{6} \mathrm{~J} / \mathrm{m}^{3}, \quad \beta=3.4 \times 10^{-3} 1 / K, \quad T_{0}^{*}-T_{1}^{*}=1 \mathrm{~K}, \quad T_{1}^{*}=300 \mathrm{~K}$

$L_{e}=500, P_{r}=5, P_{r M}=1, N_{A}=5, N_{B}=7.5 \times 10^{-4}, R_{n}=0.122$ and $M=0.5$

and it is found $F_{1}$ is positive and $F_{2}$ is negative making $-F_{2} / F_{1}$ positive, a requirement for the existence of oscillatory convection (Fig. 1). Thus, the oscillatory convection exists for alumina-water nanofluid which was nonexistent when it was saturated in a porous medium [15].

\section{Results and discussion}

To discuss the effect of various parameters, we plot the stationary convection for fixed values of the remaining parameters other than the one whose effect is to be analyzed. The stationary curves have been drawn in Figs. 2, 3, 4, 5, 6 and 7 for the critical Rayleigh number $R_{a}^{\text {st }}$ verses the nanofluid magnetic number $Q$.

The behaviour of the concentration Rayleigh number $R_{n}$ is shown in Fig. 2. It is drawn for $L_{e}=200, N_{A}=5$ and $M=0.5$ for different values of $R_{n}$. It is seen that the Rayleigh unveiled in Fig. 4. The critical Rayleigh number $R_{a}$ is plotted against nanofluid magnetic number $Q$ for different values of $L_{e}$ for fixed $N_{A}=5, R_{n}=0.1, M=0.5$. It is observed that as the value of nanofluid Lewis number $L_{e}$ increases, the value of critical Rayleigh number $R_{a}$ decreases. Hence, $L_{e}$ inhibits the stability.

Figure 5 portrays that the behaviour of Hall current parameter $M$ for $R_{n}=0.1, L_{e}=200$ and $N_{A}=5$. It shows that increment in the value of Hall current parameter $M$ leads to decrease the value of critical Rayleigh number $R_{a}$. Hence, the Hall current parameter $M$ hastens the onset of convection.

An analytic comparison of the stationary convection of a nanofluid with and without Hall currents is shown in Fig. 6. It clearly shows that in the presence of Hall currents the value of Rayleigh number falls largely. Hence, the convection sets in earlier. 
Yadav et al. [15] have discussed the Hall current effect convection when the nanofluid is saturated in a horizontal porous medium. A comparison of stationary convection for the two types of medium has been shown in Fig. 7. It is important to mention that in both the cases Rayleigh number is increasing with an increase in magnetic number but rate of increment is down when the layer is saturated in a porous medium. Thus, the presence of porous medium enhances the convection and stability is achieved in a continuous medium.

\section{Conclusion}

Bénard convection in a continuous flow medium in the presence of horizontal constant magnetic field and Hall currents has been investigated for a Newtonian nanofluid. Nanoparticle flux at the boundaries is taken to be nil. It is observed that the presence of Hall current destabilizes the stationary convection and provokes the convection. The effect of other parameters like concentration Rayleigh number, modified diffusivity ratio and nanofluid Lewis number is also destabilizing.

The comparison of the convection with the one when there are no Hall currents clearly predicts that the Hall currents are responsible to exhibit the instability of the system.

The comparison of the present configuration with the one in a porous medium says that the porous medium sets the convection earlier.

Finally, the conditional existence of oscillatory convection has been obtained. Its existence has been verified for alumina water nanofluid.

\section{Compliance with ethical standards}

Conflict of interest The authors declare that there is no conflict of interest amongst them.

Open Access This article is licensed under a Creative Commons Attribution 4.0 International License, which permits use, sharing, adaptation, distribution and reproduction in any medium or format, as long as you give appropriate credit to the original author(s) and the source, provide a link to the Creative Commons licence, and indicate if changes were made. The images or other third party material in this article are included in the article's Creative Commons licence, unless indicated otherwise in a credit line to the material. If material is not included in the article's Creative Commons licence and your intended use is not permitted by statutory regulation or exceeds the permitted use, you will need to obtain permission directly from the copyright holder. To view a copy of this licence, visit http://creativecommons. org/licenses/by/4.0/.

\section{References}

1. Choi SUS, Eastman JA (1995) Enhancing thermal conductivity of fluids with nanoparticles. ASME Fed 66:99-105

2. Yu HW, France DM, Routbort JL, Choi SUS (2009) Review and comparison of nanofluid thermal conductivity and heat transfer enhancements. J Heat Transf Eng 29:432-460. https://doi. org/10.1080/01457630701850851

3. Eastman JA, Choi SUS, Li S, Yu HW, Thompson LJ (2001) Anomalously increased effective thermal conductivities of ethylene glycol-based nanofluids containing copper nanoparticles. Appl Phys Lett 78:718-720. https://doi.org/10.1063/1.1341218

4. Buongiorno J (2006) Convective transport in nanofluids. ASME J Heat Transfer 128:240-250. https://doi.org/10.1115/1.2150834

5. Das SK, Choi SUS, Yu W, Pradeep T (2007) Nanofluids: science and technology. Willy J \& Sons, Saulnierville

6. Michaelides, Efstathios ES (2014) Nanofluidics: thermodynamic and transport properties. ISBN 978-3-319-05621-0

7. Tripathi D, Bég OA (2014) A study on peristaltic flow of nanofluids: Application in drug delivery systems. Int J Heat Mass Transf 70:61-70. https://doi.org/10.1016/j.ijheatmasstrans fer.2013.10.044

8. Wong KV, Leon OD (2010) Applications of nanofluids: current and future. Adv Mech Eng 2:1. https://doi.org/10.1155/2010/519659

9. Tzou DY (2008) Thermal instability of nanofluids in natural convection. Int J Heat Mass Transf 51:2967-2979. https://doi. org/10.1016/2007.09.014

10. Heris ZS, Salehi H, Noie HS (2012) The effect of magnetic field and Nanofluid on thermal performance of two-phase closed thermosiphon. Int J Phys Sci 7:534-543. https://doi.org/10.5897/ IJPS11.1019

11. Hamada MAA, Pop I, Ismail MdMA (2011) Magnetic field effects on free convection flow of a nanofluid past a vertical semi-infinite flat plate. Nonlinear Anal Real World Appl 12:1338-1346. https://doi.org/10.1016/j.nonrwa.2010.09.014

12. Ghasemi B, Aminossadati MS, Raisi A (2011) Magnetic field effect on natural convection in a nanofluid-filled square enclosure. Int J Therm Sci 50:1748-1756. https://doi.org/10.1016/j.ijthermals ci.2011.04.010

13. Karcher C, Kühndel J (2016) Control of heat transfer in engine coolers by Lorentz forces. J Phys Conf Ser 745:032050. https:// doi.org/10.1088/1742-6596/745/3/032050

14. Hall EH (1879) On a new action of the magnet on electric current. Am J Math 2:287-292. https://doi.org/10.2307/2369245

15. Yadav D, Mohamed RA, Cho H, Lee J (2015) Effect of Hall current on the onset of MHD convection in a porous medium layer saturated by a nanofluid. J Appl Fluid Mech 9:2379-2389. https ://doi.org/10.18869/acadpub.jafm.68.236.25048

16. Das S, Jana RN, Makinde OD (2016) Magnetohydrodynamic free convective flow of nanofluid past an oscillating porous flat plate in a rotating system with thermal radiation and Hall effects. J Mech Eng 32:197-210. https://doi.org/10.1017/jmech.2015.49

17. Wahed A, Mohamed S (2017) Rotating ferro-nanofluid over stretching plate under the effect of hall current and joule heating. J Magn Magn Mater 429:287-293. https://doi.org/10.1016/j. jmmm.2017.01.032

18. Abbasi FM, Maimoona G, Shehzad SA (2018) Hall effects on peristalsis of boron nitride-ethylene glycol nanofluid with temperature dependent thermal conductivity. Physica E Low Dimens Syst Nanostruct 99:275-284. https://doi.org/10.1016/j.physe .2018.02.006

19. Shah Z, Islam S, Gul T, Bonyah E, Khan MA (2018) The electrical MHD and Hall current impact on micropolar nanofluid flow between rotating parallel plates. Results Phys 9:1201-1214. https://doi.org/10.1016/j.rinp.2018.01.064 
20. Krishna MV, Chamkha AJ (2018) Hall effects on MHD squeezing flow of a water-based nanofluid between two parallel disks. J Porous Media 22:209-223. https://doi.org/10.1615/JPorM edia.2018028721

21. Krishna MV, Chamkha AJ (2019) Hall and ion slip effects on MHD rotating boundary layer flow of nanofluid past an infinite vertical plate embedded in a porous medium. Results Phys 15:102652. https://doi.org/10.1016/j.rinp.2019.102652

22. Shah Z, Alzahrani EO, Alghamdi W, Ullah MZ (2020) Influences of electrical MHD and Hall current on squeezing nanofluid flow inside rotating porous plates with viscous and joule dissipation effects. J Therm Anal Calorim 140:1215-1227. https://doi. org/10.1007/s10973-019-09176-7

23. Sharma J, Gupta U (2020) Nanofluid convection under Hall currents and LTNE effects. J Mater Proces 26:3369-3377. https://doi. org/10.1016/j.matpr.2019.10.149

24. Baehr HD, Stephan K (2011) Heat and mass transfer, 3rd edn. Springer, New York
25. Yadav D, Bhagrava R, Aggarwal GS (2013) Thermal instability in a nanofluid layer with vertical magnetic field. J Eng Math 80:147-164. https://doi.org/10.1007/s10665-012-9598-1

26. Nield DA, Kuznetsov AV (2014) Thermal instability in a porous medium layer saturated by a nanofluid: a revised model. Int J Heat Mass Transf 68:211-214. https://doi.org/10.1016/j.ijhea tmasstransfer.2013.09.026

27. Chandrasekhar S (1961) Hydrodynamic and Hydromagnetic stability. Oxford University Press, Oxford

Publisher's Note Springer Nature remains neutral with regard to jurisdictional claims in published maps and institutional affiliations. 